\title{
EFFICACY AND SAFETY OF GABAPENTIN AND PREGABALIN IN CHEMOTHERAPY-INDUCED PERIPHERAL NEUROPATHY
}

\author{
MANJUSHREE $\mathbf{N}^{1}$, ANANYA CHAKRABORTY ${ }^{1 *}$, SHASHIDHAR $\mathbf{V}^{2}$ \\ ${ }^{1}$ Department of Pharmacology, Vydehi Institute of Medical Sciences and Research Centre, Whitefield, Bengaluru, India. ${ }^{2}$ Department of \\ Oncology, Vydehi Institute of Medical Sciences and Research Centre, Whitefield, Bengaluru, India. Email: dr_ananya@yahoo.com
}

Received: 11 October 2020, Revised and Accepted: 30 November 2020

\section{ABSTRACT}

Objectives: Chemotherapy-Induced Peripheral Neuropathy (CIPN) occurs as a common Adverse Drug Reaction (ADR) of anti-cancer drugs. The prevalence varies from $10 \%$ to $100 \%$. To date, there is no standard effective treatment protocol for this condition. However, the neuro-modulators such as gabapentin and pregabalin are increasingly being used to treat CIPN. With this background this study was undertaken to compare the efficacy and safety of gabapentin and pregabalin in CIPN.

Methods: This study was conducted in the department of medical oncology at Vydehi Institute of Medical Sciences and Research Centre, Bengaluru. It was initiated after the approval from Institutional Ethics Committee. After obtaining written informed consent, the participants were randomized into two groups. Group A received gabapentin, $300 \mathrm{mg}$ orally and Group B received pregabalin $75 \mathrm{mg}$ orally; twice daily for 8 weeks. They were followed up at 2, 4, and 8 weeks. The intensity and quality of pain were assessed by visual analog scale (VAS) and pain quality assessment scale (PQAS). Safety was assessed by reported ADR. Data were analyzed using Student's t-test and Mann-Whitney U-test. $\mathrm{p}=0.05$ or less was considered as statistically significant.

Results: Reduction in VAS and PQAS scores at 8 weeks was statistically significant in each group ( $\mathrm{p}<0.0001)$. The ADR common to both the groups was drowsiness and sedation. The prevalence of ADR was more in the gabapentin group.

Conclusion: Both gabapentin and pregabalin have similar clinical efficacy in the treatment of CIPN. The prevalence of ADR was higher in gabapentin group compared to pregabalin group.

Keywords: Chemotherapy-induced neuropathic pain, Chemotherapy-induced peripheral neuropathy, Gabapentin, Pregabalin.

(C) 2021 The Authors. Published by Innovare Academic Sciences Pvt Ltd. This is an open access article under the CC BY license (http://creativecommons.org/ licenses/by/4.0/) DOI: http://dx.doi.org/10.22159/ajpcr.2021v14i1.39978. Journal homepage: https://innovareacademics.in/journals/index.php/ajpcr

\section{INTRODUCTION}

Chemotherapy-induced peripheral neuropathy (CIPN) is a disabling pain condition that occurs as a common adverse effect of anti-cancer drugs. In India, there are at present 2.5 million cancer cases. Of these patients, $30-50 \%$ experience CIPN while undergoing treatment for cancer $[1,2]$. The most common drugs that produce neuropathic pain are platinum agents such as cisplatin and carboplatin, taxanes like paclitaxel, Vinca alkaloids like vincristine; and bortezomib and thalidomide [3]. The prevalence of CIPN varies from $10 \%$ to $100 \%$ depending on the class of anti-cancer drugs or drug combinations used. CIPN can present as sensory symptoms in the hands and/or feet in a "stocking-glove" pattern, that is, pain, numbness, tingling, motor symptoms, cranial nerve deficits, or autonomic neuropathy. The exact pathophysiology of CIPN is not well understood. WHO recommended a three-step ladder for the use of analgesics for the treatment of pain. However, in case of neuropathic pain, response is very poor to analgesics.

At present, the anticonvulsant drugs such as gabapentin and pregabalin are considered as first-line treatment for neuropathic pain along with opioid analgesics [4-6]. Gabapentin was approved by Food and Drug Administration (FDA) in 1994 as an adjunct treatment in partial seizures. Later, it showed promising results in the treatment of chronic pain syndromes and neuropathic pain [7]. Pregabalin was approved by FDA in the year 2004. It was approved for the treatment of neuropathic pain in adults. Both the drugs bind to the $\alpha 2-\delta$ subunit of voltage gated calcium channels and decrease the release of neurotransmitter such as substance $\mathrm{P}$ and glutamate from primary afferent terminals [8]. Studies showed that pregabalin has an increased binding affinity for the $\alpha 2-\delta$ protein subunit of voltage gated calcium channels which is associated with greater analgesic activity compared with gabapentin [9]. Our literature search has shown very limited comparative studies of gabapentin with pregabalin as monotherapy in the treatment of CIPN. Hence, the present study was undertaken to compare the efficacy and safety of gabapentin and pregabalin in the treatment of CIPN.

\section{METHODS}

This randomized, open label, comparative study was conducted at the medical oncology department of Vydehi Institute of Medical Sciences and Research Centre, Bengaluru. It was conducted between January 2015 and May 2016. The study was approved by the institutional ethics committee. The following were the inclusion criteria (1) age between 18 and 60 years, (2) moderate to severe neuropathic pain, (3) completion of all the cycles of chemotherapy irrespective of the type of cancer, (4) participant suffering from at least one of the following symptoms such as burning sensation, shooting or lancinating pain, dysesthesia or allodynia, (5) informed consent, and (6) life expectancy of at least 3 months or more as judged by the oncologist. The diagnosis of neuropathic pain was based on history, clinical assessment and electrophysiological evidence based on nerve conduction study where ever it was feasible. The following were the exclusion criteria: (1) Patients with extreme difficulty in swallowing pills, (2) history of hypersensitivity to gabapentin or pregabalin, (3) neuropathy from any other type of nerve compression such as carpal tunnel or tarsal tunnel syndrome, (4) radiculopathy, spinal stenosis, brachial plexopathy, diabetic neuropathy, or neuropathic pain as a result of tumor compression, and radiation injury or surgery, (5) plasma creatinine $>1.5 \mathrm{mg} / \mathrm{mL}$, and (6) pregnant and lactating mothers. 
The participants were randomized based on computer generated numbers into two groups. Group A received tab gabapentin, $300 \mathrm{mg}$ twice daily orally, after food for 8 weeks. Group B received tab pregabalin, $75 \mathrm{mg}$ twice daily, orally after food for 8 weeks. The intensity of pain was assessed by visual analog scale (VAS), and the quality was assessed by pain quality assessment scale (PQAS). They were followed up at 2, 4, and 8 weeks after starting the drugs. At each follow-up visit, the clinical history was taken and assessment was done with the help of pain scales. The participants were given a dairy to note down the adverse drug reaction (ADR).

For rescue, oral morphine tablet, $5 \mathrm{mg}$, was permitted and the number of participants using rescue medications was noted. The investigations such as complete blood count, blood glucose levels, blood urea, and serum creatinine were estimated at $0,2^{\text {nd }}, 4^{\text {th }}$, and $8^{\text {th }}$ weeks. Electrocardiogram was taken at " 0 " week and at the end of the study. Data were analyzed using statistical analysis software version 9.1. Descriptive statistics such as mean and standard deviation for continuous variables and percentage for categorical variables was determined. Mean differences in VAS and PQAS scores between the two groups were compared using Mann-Whitney U-test since the data failed to show normality. The VAS and PQAS scores at 0 and $8^{\text {th }}$ week in the same group were compared using paired student's t-test. For all the tests, $\mathrm{p}=0.05$ or less was considered for statistical significance.

\section{RESULTS}

A total of 70 participants took part in the study. Out of them, 63 completed the study. Thirty-six participants received gabapentin (Group A) and 34 participants received pregabalin (Group B). Out of 36 participants in Group A, three were withdrawn due to development of ADR. Out of 34 participants in Group B, one lost follow-up and three were withdrawn due to development of ADR. The overall results are as follows:

\section{Demographic details and clinical profile}

The mean age in Group A was $50.6 \pm 12$ and in Group B, 53 \pm 7.6 respectively. Both groups had more of female participants (71.4\%). Out of all the participants, $36.50 \%$ were on treatment for Ca breast; $19.04 \%$ had Ca ovary; and remaining had other cancers. The most common anticancer drugs causing CIPN was inj. paclitaxel (42.85\%), followed by its combination with carboplatin. The results are shown in Table 1.

\section{Comparison of VAS scores}

The VAS scores in the two groups were compared for different weeks, as shown in Table 2 .

\section{Comparison of PQAS scores}

The PQAS scores for the two groups were compared for different weeks, as shown in Fig. 1.
The values are expressed as mean \pm SEM. Mann-Whitney U-test was used to evaluate the difference between groups. There was no significant difference in PQAS scores between the 2 groups on enrolment.

Mean reduction of VAS scores in gabapentin and pregabalin group after treatment

In Group A, VAS at " 0 " week was $8.3 \pm 1.43$ that was significantly reduced to $1.8 \pm 2.51$ at the end of treatment $(\mathrm{p}<0.0001)$. VAS in Group B was statistically reduced from $8.2 \pm 1.62$ at " 0 " week to $0.8 \pm 0.96((\mathrm{p}<0.0001)$ at the end of treatment.

Mean reduction of PQAS scores in gabapentin and pregabalin after treatment

In Group A, PQAS score significantly reduced from $34.8 \pm 6.67$ at baseline to $10.2 \pm 10.96$. In Group B, it statistically reduced from $36.9 \pm 8.5$ to $4.5 \pm 3.66$, respectively $(\mathrm{p}<0.0001)$.

Rescue medication requirement in the study groups

Rescue medications were required by 2 (6.06\%) participants in Group A, and 1 (3.33\%) participant in Group B, respectively.

\section{ADR in the study groups}

The prevalence of ADR was higher in Group A (21.1\%) when compared to Group B (16.6\%). The ADR in Group A was sedation (6.60\%), drowsiness (9.09\%), and diplopia and blurring of vision (3.03\%); and in Group B was sedation (13.3\%) and drowsiness (3.3\%) respectively. Three participants in gabapentin group were withdrawn from the study because of ADRs. Two participants developed swelling of the face and eye lids, as shown in (Fig. 2) and one participant developed excessive drowsiness and sedation. Three participants in pregabalin group were withdrawn from the study because of ADRs. One participant developed pedal edema, and other two patients developed excessive sedation.

\section{DISCUSSION}

In the present study, the prevalence of CIPN was found to be more in females $(71.4 \%)$ than males. However, the previous studies showed

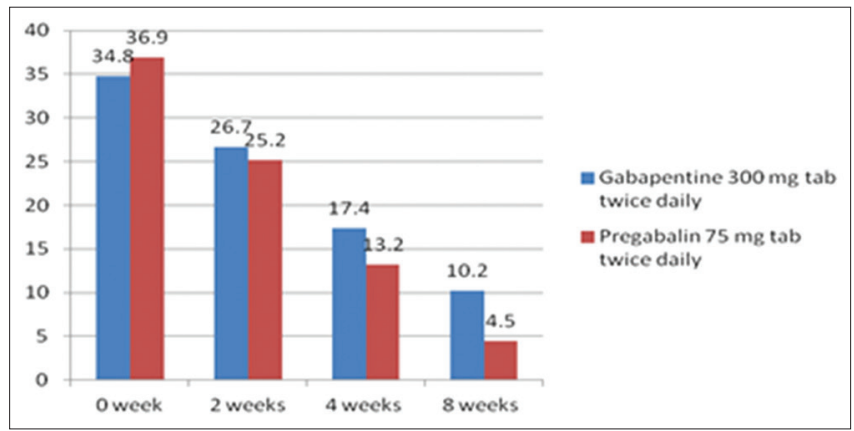

Fig. 1: Comparison of pain quality assessment scale mean scores

Table 1: Profile of disease and chemotherapy received in the groups

\begin{tabular}{llll}
\hline Diagnosis and medications & Diagnosis (n=63) \% & Group A (n=33) \% & Group B (n=30) \% \\
\hline Ca breast & $23(36.50)$ & $16(48.48)$ & $7(23.33)$ \\
Ca lung & $5(7.93)$ & $5(15.15)$ & 0 \\
Ca ovary & $12(19.04)$ & $5(15.15)$ & $7(23.33)$ \\
Ca esophagus & $3(4.76)$ & 0 & $3(10)$ \\
Multiple myeloma & $7(11.11)$ & $2(6.06)$ & $516.66)$ \\
Ca cervix & $1(1.58)$ & 0 & $1(3.33)$ \\
Others & $12(19.04)$ & $5(15.15)$ & $7(23.33)$ \\
Chemotherapy drugs & & & $13(39.39)$ \\
$\quad$ Inj Paclitaxel+inj Carboplatin & $23(36.5)$ & $17(51.51)$ & $10(33.33)$ \\
Inj paclitaxel & $27(42.85)$ & $2(6.06)$ & $5(16.66)$ \\
Inj bortezomib+cap thalidomide & $7(11.11)$ & $1(3.03)$ & $1(3.33)$ \\
Inj vincristine & $2(3.17)$ & 0 & $2(6.66)$ \\
Inj oxaliplatin & $2(3.17)$ & 0 & $2(6.66)$ \\
Inj cisplatin & $2(3.17)$ & 0 & \\
\hline
\end{tabular}

n=Total number of participants. Group A: Gabapentin, 300mg twice daily orally; Group B: Pregabalin: $75 \mathrm{mg}$ twice daily orally 
Table 2: Comparison between VAS scores between the groups

\begin{tabular}{llll}
\hline Weeks of treatment & Group A & Group B & p value \\
\hline 0 week & $8.3 \pm 1.43$ & $8.2 \pm 1.62$ & 0.487 \\
2 weeks & $6.1 \pm 1.75$ & $5.6 \pm 1.43$ & 0.336 \\
4 weeks & $3.7 \pm 2.32$ & $3.0 \pm 1.27$ & 0.202 \\
8 weeks & $1.8 \pm 2.51$ & $0.8 \pm 0.96$ & 0.234 \\
\hline
\end{tabular}

The values are expressed as mean \pm SEM. Mann-Whitney U-test was used to evaluate the difference between two groups. By assuming the level of significance as 0.05 , there was no significant difference in VAS: Visual analog scale scores. Group A: Gabapentin, 300 mg twice daily orally:

Group B: Pregabalin: 75 mg twice daily orally

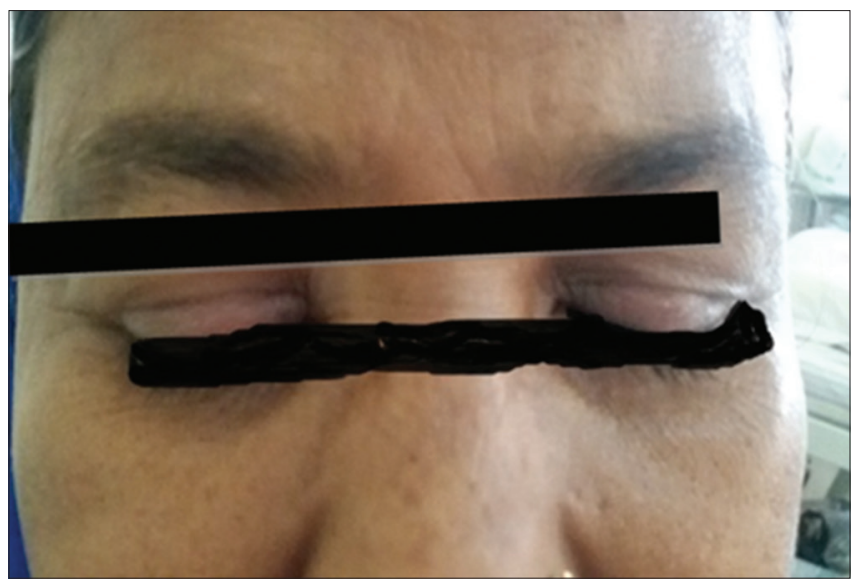

Fig. 2: Gabapentin-induced swelling of the face and eye lids

male preponderance for CIPN [7,9]. This may be because of the fact that carcinoma of breast is one of most common cancer in our hospital set up. In this study, the prevalence of CIPN was found to be highest in the age group of 51-60 years. This coincides with the previous studies [4,9]. In the present study, neuropathy was found to be highest in patients receiving paclitaxel. In the previous studies too, paclitaxel was found to be the most neurotoxic drug, followed by cisplatin, oxaliplatin, bortezomib, thalidomide, and vincristine [10]. In our study, both gabapentin and pregabalin were effective in reducing the VAS scores and PQAS scores at the end of the study ( 8 weeks) when compared to baseline scores ( 0 week). The reduction in VAS scores and PQAS scores was similar with no statistically significant difference between both the groups. This was the first study that compared the efficacy and safety of the drugs in CIPN. The previous studies conducted on the treatment of neuropathic pain demonstrated that pregabalin has significant efficacy in reducing the quality of pain than gabapentin but was no significant difference was noted in reduction of pain intensity $[11,12]$

In the present study, the common adverse effects were sedation, drowsiness, pedal edema, diplopia, and blurring of vision. The ADRs were similar to those documented in the previous studies [12-14].

Thus, by comparing the efficacy of pregabalin and gabapentin in this study, we found that both gabapentin and pregabalin have similar efficacy. Pregabalin had comparatively a smaller number of ADRs when compared to gabapentin. The number of rescue medications in gabapentin group was more compared to pregabalin group. Pregabalin may be preferred over gabapentin in view of ADRs and rescue medication requirement. The study was an open labeled study. A double-blind randomized study would have been better. A study with larger population with follow-up beyond 8 weeks would give statistical results which can be better correlated to the general population. Furthermore, nerve conduction could not be done in all patients due to economic issues. Hence, future studies with a large sample size, and long follow-up are required as both the drugs are relatively new in Indian market but have wide usage.

\section{ACKNOWLEDGMENT}

The authors are thankful to Dean, Vydehi Institute of Medical Sciences and Research Centre, Bengaluru, for the administrative support toward the study.

\section{AUTHORSHIP DETAILS}

All authors contributed to the conception, design, interpretation, and compilation of results, and execution of the study. The corresponding author drafted the manuscript, and incorporated the suggestions from other authors. The study was part of postgraduate dissertation of the first author, the second, and third authors were guide, and coguide.

\section{CONFLICTS OF INTEREST}

The authors declare that they have no conflicts of interest

\section{REFERENCES}

1. Overview: National Cancer Control Programme, India. Available from: https://www.who.int/cancer/modules/India.pdf?ua=1. [Last accessed on 2018 Dec 16]

2. Lesage P, Portenoy RK. Trends in cancer pain management. Cancer Control 1999;6:136-45.

3. Trivedi MS, Hershman DL, Crew KD. Management of chemotherapyinduced peripheral neuropathy. Am J Hematol Oncol 2015;2:4-10.

4. Banerjee M, Pal S, Bhattacharya B, Ghosh B, Mondal S, Basu B. A comparative study of efficacy and safety of gabapentin versus amitriptyline as coanalgesics in patients receiving opioid analgesics for neuropathic pain in malignancy. Indian J Pharmacol 2013;45:334-8.

5. Keskinbora K, Pekel AF, Aydinli I. Gabapentin and an opioid combination versus opioid alone for the management of neuropathic cancer pain: A randomized open trial. J Pain Symptom Manag 2007;34:183-9.

6. Toth C. Substitution of gabapentin therapy with pregabalin therapy in neuropathic pain due to peripheral neuropathy. Pain Med 2010;11:456-65.

7. Honarmand A, Safavi M, Zare M. Gabapentin: An update of its pharmacological properties and therapeutic use in epilepsy. J Res Med Sci 2011;16:1062-9.

8. Bockbrader HN, Wesche D, Miller R, Chapel S, Janiczek N, Burger P. A comparison of the pharmacokinetics and pharmacodynamics of pregabalin and gabapentin. Clin Pharmacokinet 2010;49:661-9.

9. Ghosh AK, Kundu A, Das AP, Bhattacharya KB. Comparative study of efficacy and safety of pregabalin and gabapentin in neuropathic pain. Asian J Pharm Life Sci 2012;2:64-71.

10. Argyriou AA, Kyritsis AP, Makatsoris T, Kalofonos HP. Chemotherapyinduced peripheral neuropathy in adults: A comprehensive update of the literature. Cancer Manag Res 2014;6:135-47.

11. Moulin DE, Boulanger MD, Williamson OD. Pharmacological management of chronic neuropathic pain: Revised consensus statement from the Canadian pain society. Pain Res Manag 2014;19:328-35.

12. Devi P, Madhu K, Kulkarni C. Evaluation of efficacy and safety of gabapentin, duloxetine, and pregabalin in patients with painful diabetic peripheral neuropathy. Indian J Pharmacol 2012;44:51-6.

13. Kaydok E, Levendoglu F, Ozerbil MO, Karahan AY. Comparison of the efficacy of gabapentin and pregabalin for neuropathic pain in patients with spinal cord injury: A crossover study. Acta Med Mediterr 2014;30:1343-8

14. Ghai A, Gupta M, Hooda S, Singla D, Wadhera R. A randomized controlled trial to compare pregabalin with gabapentin for postoperative pain in abdominal hysterectomy. Saudi J Anaesth 2011;5:252-7. 